\title{
Evaluation of the Disease Burden of Sporadic Creutzfeldt-Jacob Disease with the Surveillance Data from 2013 to 2017 in China
}

\section{Chen Gao}

NIVC『ChinaCDC

\section{Lina Zhang}

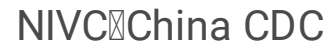

\section{Kang Xiao}

NIVC $₫$ China CDC

\section{Wei Zhou}

NIVC,Chian CDC

\section{Yuan Wang}

NIVC,China CDC

\section{Cao Chen}

NIVC,China CDC

\section{Qi Shi}

NIVC, China CDC

Xiao-Ping Dong ( $\square$ dongxp238@sina.com )

National Institute for Viral Disease Control and Prevention https://orcid.org/0000-0002-1566-8447

\section{Research Article}

Keywords: Sporadic Creutzfeldt-Jacob disease, DALY, indirect economic burden, surveillance

Posted Date: July 28th, 2020

DOI: https://doi.org/10.21203/rs.3.rs-44947/v1

License: (c) (i) This work is licensed under a Creative Commons Attribution 4.0 International License. Read Full License 


\section{Abstract}

Background Sporadic Creutzfeldt-Jacob disease (sCJD) is the commonest type of human prion disease and cause $100 \%$ fatality. The morbidity of SCJD is about 1 to 2 cases/million/year worldwide.

Methods The disease burden of SCJD and the indirect economic burdens in China were evaluated based on the data of Chinese National CJD Surveillance from 2013 to 2017.

Results The Disability-Adjusted Life Year (DALY) of SCJD was measured with 753 probable SCJD patients. The total DALY of 753 SCJD cases in the past five surveillance years was 16202.62 personyears. The Years of Life Lost (YLL) was the predominant component for DALY reaching to more than $95 \%$, while the Years Lost due to Disability (YLD) accounted for less than $5 \%$ only. The highest DALY was noticed in the group of 45-50 year-old (6541.49), followed by the groups of 60-69 year-old (6311.25). Furthermore, the indirect economic burdens of 753 SCJD patients were estimated with the official capital data. Total indirect economic burden for five years was 372.08 million RMB (1US $\$=6.9$ RMB), with the average of 494,133 RMB per case. The patients aged 45-59 year-old were the predominant population that accounted for approximate $73 \%$ of total indirect economic burden. Although still lacking of the exact annual incidence of SCJD in China, the DALY intensity of SCJD in China could be estimated and predicted as 2.2 to 4.4 person-years $/ 100,000$ with the global morbidity of SCJD.

Conclusion This is the first disease burden assay for SCJD in China, which will supply fundamental evidence for further development of medical strategy.

\section{Introduction}

Sporadic Creutzfeldt-Jacob disease (SCJD) is the commonest subtype of human prion diseases with the estimated incidence of 1 to 2 case /million/year globally (1-3). A serial of neuropsychiatric symptoms may be noticed in SCJD patients, mainly including progressive dementia, pyramidal and extrapyramidal symptoms, myoclonus, visual or cerebellar disturbance, as well as mutism in the late stage $(4,5)$. Compared with other neurodegeneration diseases, such as Alzheimer disease (AD), Parkinson disease (PD), amyotrophic lateral sclerosis (ALS), etc., SCJD usually shows rapid progression and short clinical duration. Most of the patients die within 2 years after onset and the fatality rate of SCJD is $100 \%(6,7)$, which causes great disease burden not only on the patients and their families, but also on the society.

China has started the national CJD surveillance since 2006. Up to 2018, thousands of SCJD cases and more than 150 cases of various genetic prion diseases have been diagnosed via National CJD surveillance network $(8,9)$. Like the features of SCJD globally, the prevalence of Chinese SCJD patients does not reveal any seasonal- and geographic-association. The most frequent onset age is in the group of 60-69 year-old $(y)$. The median survival times after onset is estimated as 7 months (6).

Disability-Adjusted Life Year (DALY) is measurement of the gap between current health status and an ideal health situation, which has been widely used by countries in the evaluation of the burden of many 
diseases, including neurodegenerative disorders. However, only a few reports have addressed the DALYs of SCJD, and so far as we know, there is still lacking of the relevant data for SCJD in China. In this study, by using the data of national CJD surveillance from 2013 to 2017, we evaluated and calculated the DALYs based on 753 Chinese probable SCJD patients, and further estimated the indirect economic burden of SCJD.

\section{Materials And Methods}

\section{Data collection}

All information of the SCJD patients came from the National Center for CJD surveillance network, China $\mathrm{CDC}$. The reporting and diagnostic procedures for various human prion diseases within National CJD surveillance network were described elsewhere (9). In this study, after excluding other diagnosed cases, we recruited all probable-diagnostic SCJD patients from 2013 to 2017, totally 753 cases. The death times for the patients were either via the follow-up survey or via accessing into the Disease Surveillance Points System. The national population data and Gross domestic product (GDP) data are derived from the statistical yearbook of the Chinese National Bureau of Statistics (10). According to the Coale and Demeny West Level 26 Life Table, the life expectancy of women at birth is 82.5 years, and that of men at birth is 80.0 years.

\section{Calculation of DALY for SCJD}

DALYs for a disease or health condition are calculated as the sum of the Years of Life Lost (YLL) due to premature mortality in the population and the Years Lost due to Disability (YLD) for people living with the health condition or its consequences. One DALY means a total healthy year lost. The calculations for DALY, YLL and YLD of SCJD were conducted according to the protocols described elsewhere $(11,12)$. Disability weights were extracted based on surveys of the experts who had a basic knowledge about prion (13).

\section{Calculation of indirect economic burden for SCJD}

Indirect economic burden $=$ productivity weight $\times$ DALY $\times$ GDP per capita $(14,15)$. The productivity weight varies among the different age groups. According to the published data (16), the productivity weights of different age groups were 0.15 for the group of $0-14$ year-old $(y), 0.75$ for the group of $15-44 y, 0.80$ for the group of $45-59 \mathrm{y}$, and 0.1 for the group above $60 \mathrm{y}$.

\section{Statistical analysis}

The database was established by Microsoft Excel 2016. A descriptive analysis was applied. ArcGIS 10.3 was used to draw thematic map of Chinese SCJD patients' distribution. T-test was applied to analyze whether there were differences in DALY caused by sex.

\section{Results}


Totally, 753 sCJD patients diagnosed in the National Center for CJD surveillance network from 2013 to 2017 were recruited in this study. The case numbers from 2013 to 2017 were 108, 134, 133, 159 and 219, respectively. Up to the end of August 2018, there were 45 patients referred in 2017 still alive. The gender ratio was 1:0.9 (M:F). Majority of the patients was in the groups of 60-69 y (325, 44.2\%), 45-59 y (247, $33.6 \%)$ and $70-79$ y $(132,18.0 \%)$. Only small portions of the SCJD patients were younger than $45 \mathrm{y}(31$, $4.2 \%)$ or older than $80 \mathrm{y}(18,2.4 \%)$. Based on the permanent addresses, large portions of the patients were from the eastern and middle part of China.

\section{DALYs for SCJD}

The losses of YLL, YLD of SCJD patients each year were calculated, and then, the DALYs of SCJD patients each year were generated. As shown in Table 1, the total DALY of 753 SCJD cases in the past five years was 16202.62 person-years, showing an increased tendency along with years, which was directly due to the increased numbers of the diagnosed SCJD cases. Analysis of the components of DALYs revealed that YLLs were obviously predominant, reaching to more than 95\%, while YLD accounted for less than $5 \%$ only.

Table 1

DALYs of sCJD patients from 2013 to 2017

\begin{tabular}{|llllll|}
\hline Year & Case number & DALY & YLL & YLD & YLL/DALY(\%) \\
\hline 2013 & 108 & 2520.959 & 2421.599 & 99.360 & 96.1 \\
\hline 2014 & 134 & 3127.607 & 3006.167 & 121.440 & 96.1 \\
\hline 2015 & 133 & 2789.225 & 2666.865 & 122.360 & 95.6 \\
\hline 2016 & 159 & 3663.414 & 3517.134 & 146.280 & 96.0 \\
\hline 2017 & 219 & 4101.412 & 3899.932 & 201.480 & 95.1 \\
\hline Total & 753 & 16202.616 & 15511.696 & 690.920 & 95.7 \\
\hline
\end{tabular}

The distributions of DALY of 753 SCJD patients in five years among the different age groups were further analyzed. As shown in Fig. 1, the highest DALY was noticed in the group of 45-50 y (6541.49), followed by the groups of 60-69 y (6311.25). Total DALYs decreased apparently in the groups of 70-79 y (1594.42) and 30-44 y (1342.22), and became much less in the senior group (238.09) and young group (175.14) (Fig. 2). Subsequently, the gender was introduced into the evaluation. Those data highlight that the patients aged 45-69 y contribute mainly the DALYs and undertake the main disease burden. Life loss is the deterministic factor for the DALYS of SCJD patients.

\section{Indirect economic burdens for SCJD}

To estimate the indirect economic burden of Chinese SCJD patients, the official capital data were introduced. The estimated indirect economic burdens of SCJD were summarized in Table 2. Total indirect 
economic burden for five years was 372.08 million RMB (1US \$=6.9 RMB). The average indirect economic burden per case was 494,133 RMB. As expected, the indirect economic burden each year revealed an increased trend along with years because of the increase of SCJD cases, meanwhile, did not illustrate significant difference between male and female patients by different years $(t=-0.967, P=0.362)$.

Table 2

The estimated indirect economic burden of SCJD patients from 2013 to 2017

\begin{tabular}{|lllll|}
\hline Year & Male & Female & Total & Per case \\
\hline 2013 & 20393184.47 & 31600082.91 & 51993267.38 & 481419.14 \\
\hline 2014 & 43344742.26 & 25117569.03 & 68462311.28 & 510912.77 \\
\hline 2015 & 29093147.59 & 27349855.36 & 56443002.96 & 424383.48 \\
\hline 2016 & 37211143.41 & 49562003.02 & 86773146.43 & 545743.06 \\
\hline 2017 & 51525375.83 & 56885517.08 & 108410892.91 & 495026.91 \\
\hline Total & 181567593.57 & 190515027.39 & 372082620.96 & 494133.63 \\
\hline
\end{tabular}

The indirect economic burdens in various age groups were estimated further. As expected, the patients in the age of 45-59 y were the predominant population for indirect economic burden, causing 271.49 million RMB losses that accounted for approximate $73 \%$ total indirect economic burden (Fig. 3). Despite of obviously fewer case numbers $(n=28)$, the patients in the group of 30-44 y contributed to more indirect economic burdens (51.73 million RMB) than those in the group of $60-69$ y (32.71 million RMB) with the most case numbers $(n=325)$.

\section{Discussion}

In this study, we have for the first time calculated the DALYs of Chinese SCJD based on 753 probable SCJD cases from 2013 to 2017. Overall, there are 16202.62 DALYs for 753 sCJD cases from 2013-2017, with the average annual loss of 3240.52 DALYs. Patients with the ages from 45 to 69 are most affected populations, contributing to $79.3 \%$ of the DALYs. Moreover, YLLs each surveillance year contribute over $95 \%$ of individual annual DALY, reflecting, from another point of view, a rapid progression and short duration of SCJD.

It is well known that if the clinical course of a disease is relatively stable, the results based on the numbers of disease using are close to that of the numbers of morbidity. In general, the exact data of morbidity are relatively difficult to obtain, while the data of the people with a special disease are much easy to be acquired. We have to confess that the numbers of SCJD in this study are far below the expected morbidity, which is hard to represent the accrual DALYs of SCJD in China. However, the morbidity of SCJD is fairly stable worldwide, which maintains 1 to 2 case /million /year (17). Although the calculated incidences of SCJD in some European countries show increasing trend during the past decade, the enhanced surveillance capacities are believed to be the reason $(18,19)$. In fact, based on our 
10 years CJD surveillance data in Beijing, the incidence is close to 1 case/million/year(20). Taking this morbidity data and the population in China, the annual case numbers of SCJD in China should be 1400 to 2800. Subsequently, according to the DALYs of 753 sCJD cases in this study, the average annual DALY of SCJD is estimated as 30124.36 to 60248.71 person-years. With the average population for the five years from 2013 to 2017 as the denominator, the DALY intensity of SCJD in China would be predicted as 0.022 to 0.044 person-years per 1,000 population, or 2.2 to 4.4 person-years per 100,000 populations.

By reviewing the published data, we have compared the predicted DALY intensity of SCJD in China with other neurodegeneration diseases, malignant tumors and some infectious diseases in China (Table 3). As expected, $A D$ and many malignant tumors, e.g., lung cancer, liver cancer, stomach carcinoma and pancreas cancer possess much high DALYs. The DALY of SCJD seems to be comparable with those of multiple sclerosis (3.32 person-years/100,000) and myasthenia gravis (2.23 person-years $/ 100,000)$. Compared with infectious diseases, the DALY of SCJD is obviously lower than that of TB, AIDS, syphilis and hepatitis B, similar as rabbis, and higher than that of hepatitis C, measles and Shigella dysentery. It indicates that although the morbidity of SCJD is low, its $100 \%$ fatality cause relatively high DALY. 
Table 3

Comparison of the DALY intensities of different diseases

\begin{tabular}{|ll|}
\hline Disease & DALY intensity (person-years /100,000) \\
\hline Alzheimer disease & 503 \\
\hline Lung cancer & 462 \\
\hline Liver cancer & 325 \\
\hline Stomach cancer & 96 \\
\hline Lung tuberculosis & 82.3 \\
\hline Pancreas carcinoma & 26.23 \\
\hline AlDS & 8.2 \\
\hline Syphilis & 7.7 \\
\hline Hepatitis B & 6.0 \\
\hline sCJD & $4.4-2.2$ \\
\hline Multiple sclerosis & 3.32 \\
\hline myasthenia gravis & 2.23 \\
\hline Rabbis & 2.2 \\
\hline Hepatitis C & 0.3 \\
\hline Measles & 0.3 \\
\hline Shegella dysentery & 0.2 \\
\hline
\end{tabular}

The total indirect economic burden of 753 SCJD patients from five years is estimated to be 372.08 million RMB. SCJD usually occurs in the older people. 475 of 753 SCJD patients (64.6\%) in this study are over 60 year-old, but account for only $11.4 \%$ of indirect economic burdens. It is understandable since we have set in this study that the main labor force for society production is the population from 14 to $59 \mathrm{y}$. However, more and more senior persons over 60 years old continue to work in China, particularly in the central cities. In addition, the retirement age in China probably will be postponed from 60 to 65 year-old. Those factors will influence the estimation of indirect economic burdens of diseases, especially the diseases involving in elder population.

\section{Conclusion}

The disease burden of SCJD and the indirect economic burdens in China were evaluated based on the data of Chinese National CJD Surveillance from 2013 to 2017. There are 16202.62 DALYs for 753 sCJD cases from 2013-2017, with the average annual loss of 3240.52 DALYs. The total indirect economic 
burden of 753 sCJD patients from five years is estimated to be 372.08 million RMB, with the average of 494,133 RMB per case.This is the first disease burden assay for SCJD in China, which will supply fundamental evidence for further development of medical strategy.

\section{Declarations}

\section{Ethical Approval and Consent to participate}

The authors assert that all procedures contributing to this work comply with the ethical standards of the relevant national and institutional committees on human experimentation and with the Helsinki Declaration of 1975, as revised in 2008. This study was approved by Ethical Review Committee of National Institute for Vial Disease Prevention and Control, China CDC. Following the principle of informed and voluntary, all signed informed consents were collected and stored by the China CJD Surveillance Center.

\section{Consent for publication}

Written informed consent for publication was obtained from all participants.

\section{Availability of Data and Materials}

The data and material supporting the findings could be obtained from Xiaoping Dong, email dongxp238@sina.com.

\section{Competing interests}

The authors declare that they have no competing interests.

\section{Fundings}

This work was supported by Chinese National Natural Science Foundation Grants $(81630062,81572048)$; Research Project (2016YFC1202700, 2017YFC1200500) and SKLID Development Grant (2015SKLID 503 and 2016SKLID603).

\section{Authors' contributions}

CG, LZ and XPD designed the study. KX and WZ collected the data. YW and CC analyzed the available data. CG and LZ prepared the manuscripts. QS and XPD reviewed and edited the manuscript. All authors read and approved the final manuscript. 


\section{Acknowledgements}

We thank all involved SCJD patients and the families for receiving our invitation participating in this study.

\section{Authors' information}

Chen Gao PhD, professor in National Institute for Viral Diseases Control and Prevention, China CDC, participate in the work of CJD surveillance in China.

Lina Zhang, a master student in National Institute for Viral Diseases Control and Prevention, China CDC.

\section{References}

1. Will RG, Ironside JW. Sporadic and Infectious Human Prion Diseases. Cold Spring Harb Perspect Med. 2017;7(1).

2. Holman RC, Belay ED, Christensen KY, Maddox RA, Minino AM, Folkema AM, et al. Human prion diseases in the United States. PLoS One. 2010;5(1):e8521.

3. Klug GM, Boyd A, Lewis V, Douglass SL, Argent R, Lee JS, et al. Creutzfeldt-Jakob disease: Australian surveillance update to December 2005. Commun Dis Intell Q Rep. 2006;30(1):144-7.

4. Belay ED, Holman RC, Schonberger LB. Creutzfeldt-Jakob disease surveillance and diagnosis. Clin Infect Dis. 2005;41(6):834-6.

5. Knight R. Infectious and Sporadic Prion Diseases. Prog Mol Biol Transl Sci. 2017;150:293-318.

6. Chen C, Wang JC, Shi Q, Zhou W, Zhang XM, Zhang J, et al. Analyses of the survival time and the influencing factors of chinese patients with prion diseases based on the surveillance data from 2008-2011. PLoS One. 2013;8(5):e62553.

7. Lundberg PO. Creutzfeldt-Jakob disease in Sweden. J Neurol Neurosurg Psychiatry. 1998;65(6):83641.

8. Gao C, Shi Q, Tian C, Chen C, Han J, Zhou W, et al. The epidemiological, clinical, and laboratory features of sporadic Creutzfeldt-Jakob disease patients in China: surveillance data from 2006 to 2010. PLoS One. 2011;6(8):e24231.

9. Shi Q, Zhou W, Chen C, Gao C, Xiao K, Wang J, et al. Quality evaluation for the surveillance system of human prion diseases in China based on the data from 2010 to 2016. Prion. 2016;10(6):484-91.

10. NBS. China statistical yearbook [Available from: http://www.stats.gov.cn/.

11. WHO

WHO. Metrics. Disability-Adjusted Life Year (DALY) [Available from: https://www.who.int/healthinfo/global_burden_disease/metrics_daly/en/. 
12. Murray CJ. Quantifying the burden of disease: the technical basis for disability-adjusted life years. Bull World Health Organ. 1994;72(3):429-45.

13. Zhang LN, Wang Y, Xiemusiye Wulayin, Xiao K, Wang J, Zhou W, et al. Assessment of disability level and weight of sporadic Creutzfeldt-Jakob disease cases in China(in Chinese). Disease surveillance. 2019;34:122-6.

14. Ji XQ, Zhou JY, H RQ. M. W, Y. X. Study on the disease burden and indirect economic burden caused by diabetes mellitus in residents of Jiangsu province, China. Chinese Journal of Prevention Control of Chronic Diseases. 2014;22:5-8.

15. Y F, F K. Z.W. Z. Life Lost and Indirect Economic Burden Caused by Diabetes in China. Chinese Health Economics. 2011;30:73-4.

16. Barnum $\mathrm{H}$. Evaluating healthy days of life gained from health projects. Soc Sci Med. 1987;24(10):833-41.

17. Chen C, Dong XP. Epidemiological characteristics of human prion diseases. Infect Dis Poverty. 2016;5(1):47.

18. Puopolo M, Catelan D, Capellari S, Ladogana A, Sanguedolce A, Fedele A, et al. Spatial Epidemiology of Sporadic Creutzfeldt-Jakob Disease in Apulia, Italy. Neuroepidemiology. 2019:1-7.

19. Feketeova E, Jarcuskova D, Janakova A, Rozpravkova E, Cifrakova Z, Farkasova-Inaccone S, et al. Creutzfeldt-Jakob disease surveillance in Eastern Slovakia from 2004 to 2016. Cent Eur J Public Health. 2018;26(Suppl):37-41.

20. Shi Q, Zhang XC, Zhou W, Xiao K, Chen C, Zhang HY, et al. Analysis of the advantage features of Beijing surveillance network for Creutzfeldt-Jakob disease. Prion. 2015;9(4):304-14.

\section{Figures}




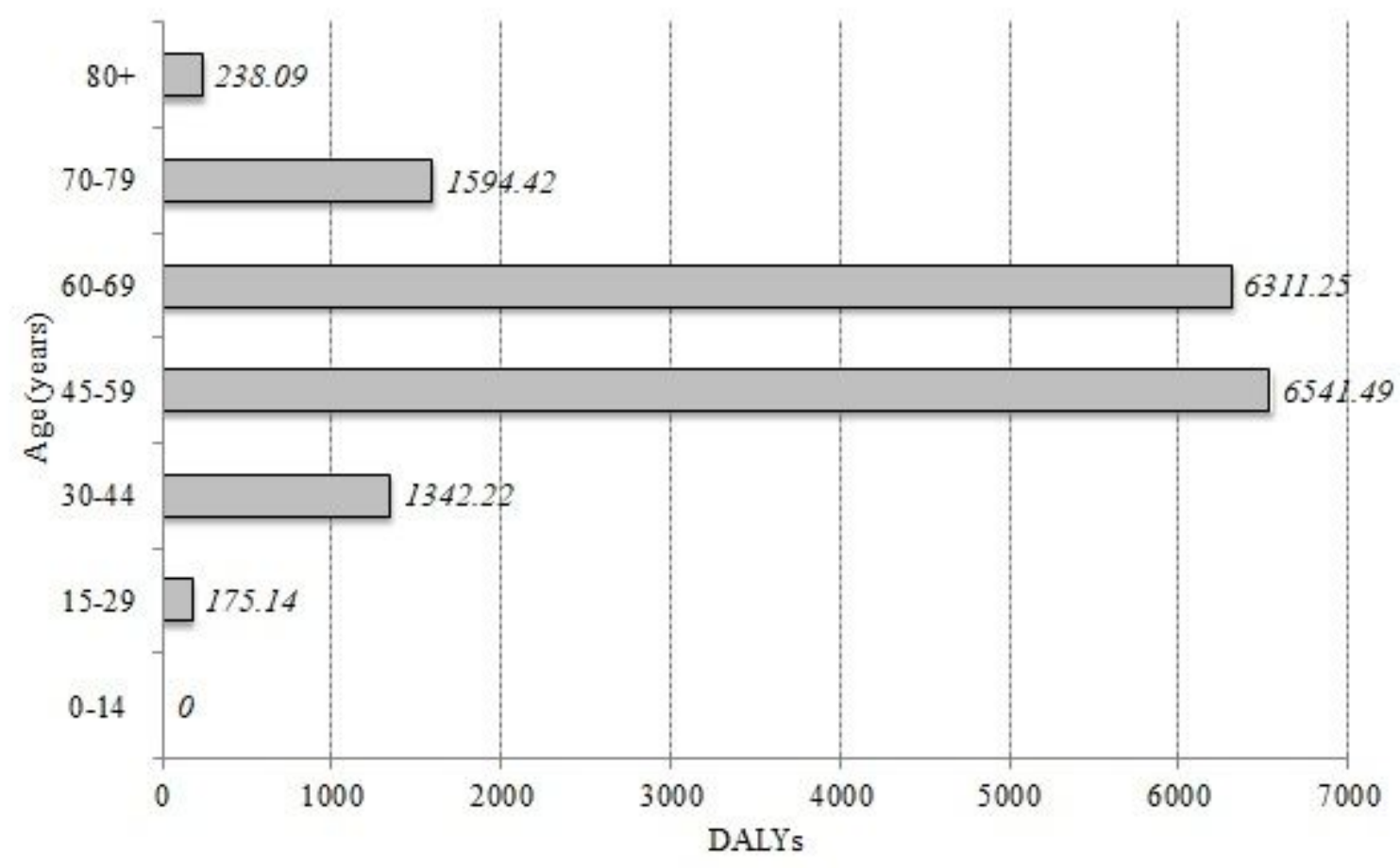

Figure 1

DALYs caused by the SCJD patients in different age groups. DALYs are showed in $X$ axis and the age groups are showed in $\mathrm{Y}$ axis. 


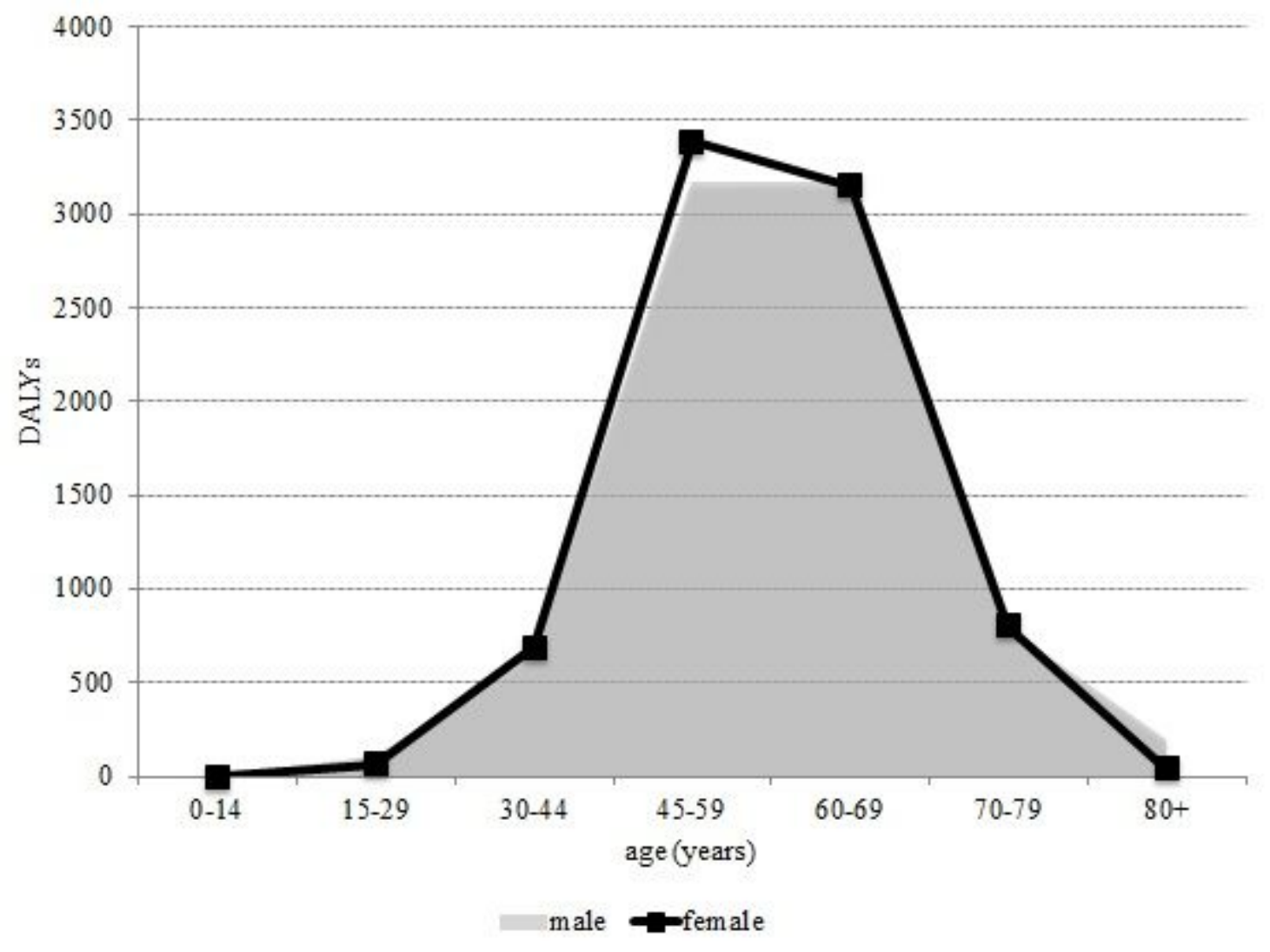

Figure 2

DALYs caused by male and female sCJD patients in different age groups. DALYs are indicated in Y axis and the age groups are showed in $\mathrm{X}$ axis. 


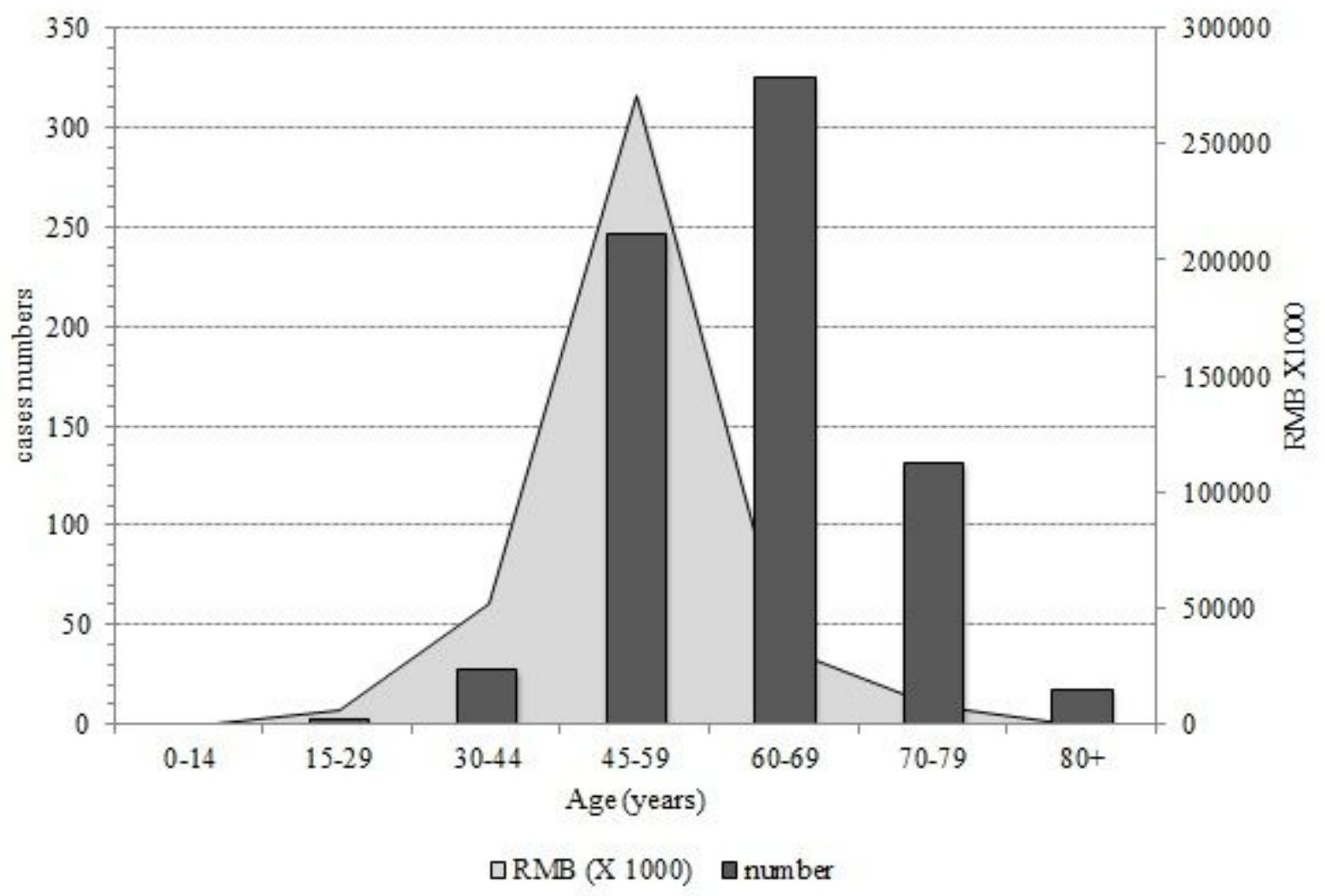

Figure 3

Indirect economic burdens of SCJD patients in different age groups. Case numbers in different age groups are showed in the left $Y$ axis and the indirect economic burdens are showed in the right $Y$ axis. The age groups are indicated in $\mathrm{X}$ axis.

\section{Supplementary Files}

This is a list of supplementary files associated with this preprint. Click to download.

- fig4.jpg 